Nota Científica

\title{
Uso de Linepithema humile Mayr, 1868 (Hymenoptera: Formicidae) como agente biológico para la limpieza de osamenta de Philodryas chamissonis (Wiegmann, 1834) (Squamata: Colubridae)
}

Use of Linepithema humile Mayr, 1868 (Hymenoptera: Formicidae) as a biological agent for cleaning the skeleton of Philodryas chamissonis (Wiegmann, 1834) (Squamata: Colubridae)

\author{
Diego Jara ${ }^{1}$ y Francisco Urra ${ }^{2}$
}

${ }^{1}$ Taller de Taxidermia, Área de Exhibiciones, Museo Nacional de Historia Natural, Casilla 787, Santiago, Chile. E-mail: diego.jara@mnhn.gob.cl. 2Área de Entomología, Museo Nacional de Historia Natural, Casilla 787, Santiago, Chile. 道*francisco.urra@mnhn.gob.cl

\section{ZooBank: urn:lsid:zoobank.org:pub:F8EA9115-6AC6-4325-9C3A-715C7944E510} https: / / doi.org/10.35249/ rche.47.2.21.22

Resumen. Se documenta por primera vez el uso de la hormiga argentina, Linepithema humile Mayr, 1868 como agente limpiador de osamenta de culebra de cola larga, Philodryas chamissonis (Wiegmann, 1834). Tras la remoción de la piel, principales paquetes musculares y vísceras, la osamenta de la serpiente fue puesta en un dispositivo que contenía un cebo basado en sacarosa. La limpieza completa de las piezas se logró luego de tres meses a temperatura ambiente en el exterior.

Palabras clave: Esqueleto; hormiga; museología; osteotecnia; serpiente.

Abstract. The use of the Argentine ant, Linepithema humile Mayr, 1868 as a cleaning agent for the longtailed snake skeleton, Philodryas chamissonis (Wiegmann, 1834) is documented for the first time. After removal of the skin, main muscle bundles and viscera, the snake's skeleton was placed in a device containing a sucrose-based bait. The complete cleaning of the pieces was achieved after three months at outdoor air temperature.

Key words: Ant; museology; osteotechnique; skeleton; snake.

Los huesos individuales y los esqueletos completos sirven como material didáctico en las escuelas y universidades, como objetos de estudio en investigaciones científicas y como piezas de exhibición en museos (Ajayi et al. 2016; Kempa et al. 2016). El uso de piezas óseas en descripciones morfológicas y estudios morfométricos sólo puede realizarse después de su adecuada preparación (Kempa et al. 2016). Para tal propósito, existen diversas técnicas, desde la simple descomposición del cadáver, hasta el uso de agentes químicos y biológicos en condiciones controladas (Aggarwal et al. 2016; Kempa et al. 2016; Lai et al. 2015; Onwama et al. 2012).

La limpieza de los huesos puede realizarse mediante maceración por acción bacteriana en agua tibia o bien, por acción de detergentes y enzimas (Lai et al. 2015); también pueden usarse pequeños animales necrófagos, como crustáceos marinos o larvas de insectos, pues la microfauna necrófaga forma parte del proceso natural de descomposición y puede

Recibido 7 Mayo 2021 / Aceptado 1 Junio 2021 / Publicado online 25 Junio 2021

Editor Responsable: José Mondaca E. 
encontrarse alimentándose sobre cadáveres de vertebrados en condiciones naturales (Kreitlow 2010). Entre los insectos, se han utilizado distintas especies, como las larvas del gusano de la harina, Tenebrio molitor (Linnaeus, 1758) (Allen y Neill 1950); las larvas de polilla de la ropa, Tineola bisselliella (Hummel, 1823) (Banta 1961); la hormiga de fuego, Solenopsis invicta Buren, 1972 (Crawford y Atkinson 1975) y larvas de moscas del género Calliphora Robineau-Desvoidy, 1830 (Majeed 2009); sin embargo, el método más extendido es el uso de las larvas de escarabajos derméstidos (Coleoptera: Dermestidae), en particular las de Dermestes maculatus De Geer, 1774.

La limpieza con larvas de Dermestes maculatus, es un método que se aplica generalmente en animales de pequeño tamaño; su principal ventaja es la limpieza económica y cuidadosa (Sommer y Anderson 1974), por lo que se considera uno de los mejores métodos para la preparación de este tipo de material (Borell 1938; Hall y Russell 1933). Los derméstidos son capaces de limpiar eficientemente los huesos en un corto período de tiempo y las piezas obtenidas quedan en condiciones de ser usadas en posteriores análisis; esto es especialmente útil con aquellas partes del esqueleto más difíciles de limpiar, tales como la columna vertebral o el cráneo; sin embargo, este proceso requiere de constante atención para evitar que las partes más frágiles sean destruidas por la actividad de los insectos (Banta 1961; Hefti et al. 1980). Después de la limpieza, los huesos pueden congelarse a $-18{ }^{\circ} \mathrm{C}$ para eliminar los insectos restantes (Dumitru et al. 2013). Otras especies de derméstidos, que también han sido usadas, son Anthrenus varius Fabricius, 1792 y A. scrophulariae (Linnaeus, 1758), las que serían recomendables para la limpieza de huesos delicados (Vorhies 1948).

El objetivo de esta nota es documentar el proceso de preparación y esqueletización de un ejemplar de culebra de cola larga, Philodryas chamissonis (Wiegmann, 1834) (Squamata: Colubridae) (Ccl 01, SAG Res. Ex. N²602 / 2018), mediante la acción de la hormiga argentina, Linepithema humile Mayr, 1868 (Hymenoptera: Formicidae). El ejemplar fue recolectado sin vida, fresco y sin lesiones aparentes, en la comuna de Pirque, Región Metropolitana de Santiago (Fig. 1a). Cabe señalar que para la recolección y tenencia de este tipo de material biológico se debe contar con una resolución emitida por el Servicio Agrícola y Ganadero de Chile (SAG), debido a que esta especie de reptil está protegida por la Ley de Caza.

La experiencia se llevó a cabo entre septiembre y diciembre de 2019. El ejemplar fue eviscerado, se removió la piel y los principales paquetes musculares, y se pasó un alambre por el canal medular de la columna vertebral, para mantener su integridad durante la acción de las hormigas. Se preparó un dispositivo fabricado con un tubo de PVC-U de 20 mm de diámetro x $1 \mathrm{~m}$ de largo, abierto por uno de sus costados; se introdujo el ejemplar por el costado y se cubrió con abundante azúcar de mesa (sacarosa) (Fig. 1b). Finalmente, se dispuso el tubo en las cercanías de una colonia de hormiga argentina para que se alimentasen de los restos, y, por consiguiente, realizaran la limpieza de los huesos.

Durante el proceso de limpieza no se observó proliferación de bacterias ni hongos, lo que podría deberse a las propiedades higroscópicas del azúcar de mesa y a la acción misma de las hormigas. Después de tres meses, el trabajo de las hormigas resultó meticuloso, entregando un ejemplar desprovisto de toda la musculatura y otros tejidos blandos (Figs. 1c, 1d). Finalmente, se realizó la limpieza general del esqueleto y sus elementos fueron ensamblados mediante pegamento instantáneo de contacto.

Comentarios. El método expuesto tiene las siguientes ventajas: a) es de bajo costo, requiere de pocos cuidados y no es necesario mantener a los insectos confinados en habitáculos; b) las hormigas argentinas están disponibles de forma natural y tienen una amplia distribución en Chile (Artigas 1994); y c) la limpieza de las piezas es meticulosa y sin daño aparente. La principal desventaja de esta técnica sería el tiempo que demora, cerca de tres meses aproximadamente, considerando que se realizó durante la primavera. Esta técnica difiere de la que emplea a la hormiga de fuego, Solenopsis invicta, pues esta última 
no requiere de un atrayente dulce, salvo que las piezas óseas estén resecas, en cuyo caso se recomienda cubrir con miel (Crawford y Atkinson 1975). En relación con los cuidados de la faena, la eficiencia de la limpieza, y el tiempo que demora el proceso, es comparable con el uso de la polilla de la ropa, Tineola bisselliella; sin embargo, esta última técnica agrega los inconvenientes de contaminación con seda producida por las larvas y la desarticulación de las pequeñas piezas, por el consumo del tejido conectivo (Banta 1961).

El uso de hormigas argentinas para la limpieza de piezas óseas sería una técnica que contribuiría al desarrollo de la osteotecnia clásica, para la preparación de pequeños esqueletos con fines de investigación o exhibición.

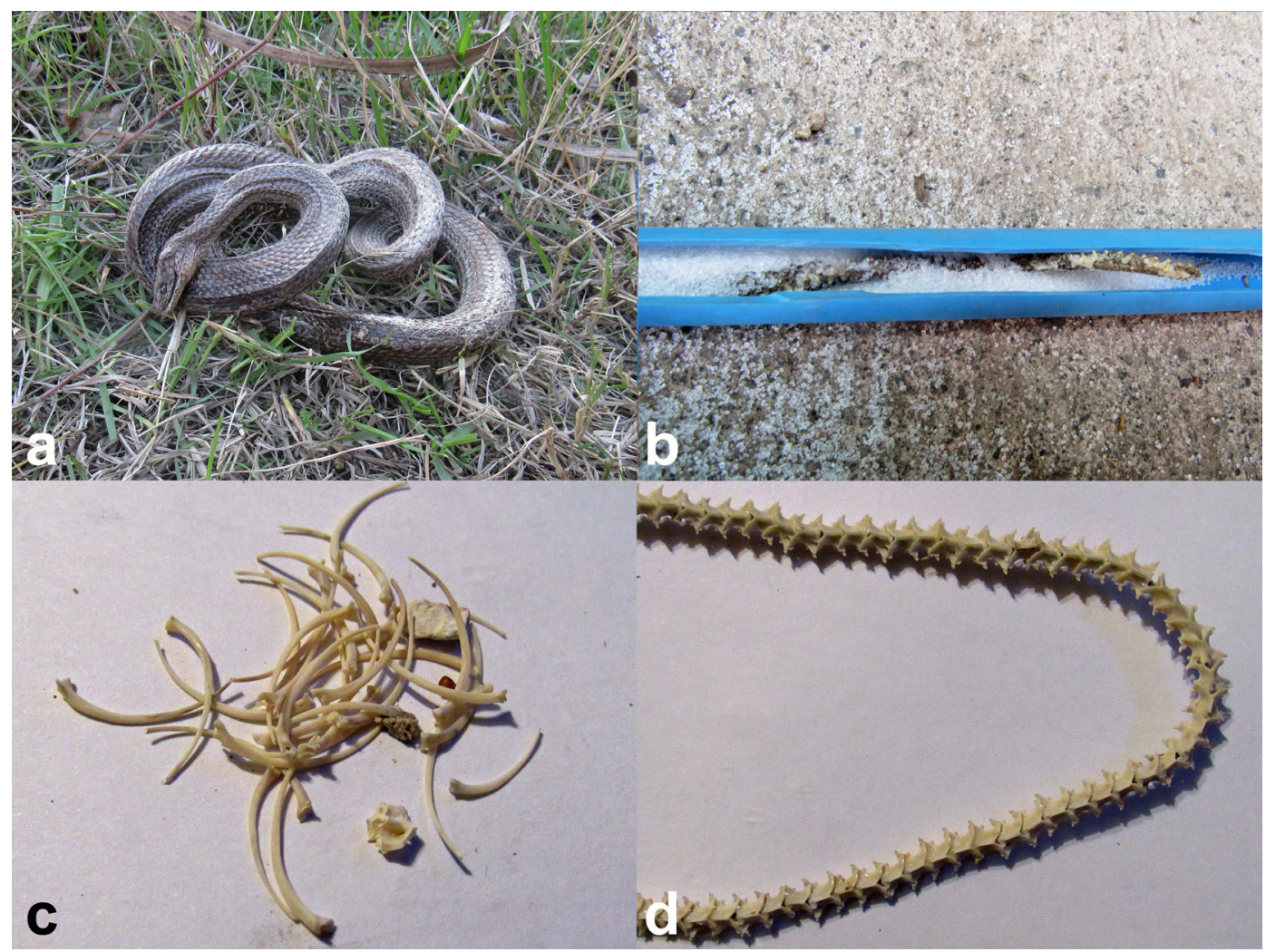

Figura 1. Proceso de limpieza de esqueleto de culebra de cola larga. a.) Ejemplar recolectado. b) Dispositivo con el ejemplar y el cebo de azúcar. c) y d) Esqueleto limpio. / Long-tailed snake skeleton cleaning process. a.) Collected specimen. b) Device with the specimen and sugar bait. c) and d) Clean skeleton.

\section{Literatura Citada}

Aggarwal, N., Gupta, M., Goyal, P.K. y Kaur, J. (2016) An alternative approach to bone cleaning methods for anatomical purposes. International Journal of Anatomy and Research, 4(2): 2216-2221.

Ajayi, A., Edjomariegwe, O. y Iselaiye, O.T. (2016) A review of bone preparation techniques for anatomical studies. Malaya Journal of Biosciences, 3(2): 76-80.

Allen, E.R. y Neill, W.T. (1950) Cleaning mammal skeletons with meal worms. Journal of Mammalogy, 31(4): 464.

Artigas, J.N. (1994) Entomología Económica. Ediciones Universidad de Concepción; Concepción, Chile, Vol. II. 943 pp. 
Banta, B.H. (1961) The use of clothes moth larvae (Lepidoptera: Tineidae) to prepare osteological specimens, with an annotated bibliography on the use of other arthropods for vertebrate skeletal preparation. The Wasmann Journal of Biology, 19(2): 265-268.

Borell, A.E. (1938) Cleaning small collections of skulls with dermestid beetles. Journal of Mammalogy, 19(1): 102-103.

Crawford, R.L. y Atkinson, J.B. (1975) Fire ants used in skeletal preparations. The Florida Entomologist, 58(1): 8.

Dumitru, I., Trăncă, S., Martonoş, C., Silaghi, F., Tuns, F., Irimescu, I. y Damian, A. (2013) Study regarding two methods of processing and preserving bird skeletons. Bulletin UASVM, Veterinary Medicine, 70(1): 66-71.

Hall, E.R. y Russell, W.C. (1933) Dermestid beetles as an aid in cleaning bones. Journal of Mammalogy, 14(4): 372-374.

Hefti, E., Trechsel, U., Rfifenacht, H. y Fleisch, H. (1980) Use of dermestid beetles for cleaning bones. Calcified Tissue International, 31: 45-47.

Kempa, K., Kulawik, M., Bartyzel, B.J., Jakubowski, M., Skubis, J. y Koczoń, P. (2016) Characterization of selected techniques of maceration bones of Gallus gallus domesticus. Folia Pomeranae Universitatis Technologiae Stetinensis, 328(39)3: 109-116.

Kreitlow, K.L.T. (2010) Insect Succession in a Natural Environment, pp. 251-270. En: Byrd, J. H. y Castner, J. L. (Eds.). Forensic Entomology, The Utility of Arthropods in Legal Investigations. Second Edition. Taylor \& Francis Group, Boca Raton, Florida, EUA. 681 pp.

Lai, P.S., Khoo, L.S., Mohd Hilmi, S., Ahmad Hafizam, H., Mohd Shah, M., Nurliza, A. y Nazni, W.A. (2015) Effectiveness of bone cleaning process using chemical and entomology approaches: time and cost. Malaysian Journal of Pathology, 37(2): 123-135.

Majeed, Z.Z. (2009) Maceration of delicate osteological material by fly larvae. Journal of Animal and Veterinary Advances, 8(11): 2147-2149.

Onwuama, K.T., Salami, S.O., Ali, O. y Nzalak, J.O. (2012) Effect of different methods of bone preparation on the skeleton of the African giant pouched rat (Cricetomys gambianus). International Journal of Morphology, 30(2): 425-427.

Sommer, H.G. y Anderson, S. (1974) Cleaning skeletons with dermestid beetles-two refinements in the method. Curator: The Museum Journal, 17(4): 290-298.

Vorhies, C.T. (1948) A chest for dermestid cleaning of skulls. Journal of Mammalogy, 29: 188-189. 